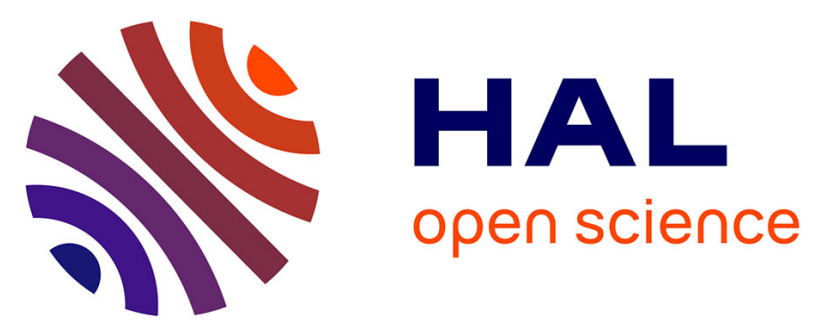

\title{
Structure-function relationships in reconstituted HDL: Focus on antioxidative activity and cholesterol efflux capacity
}

\author{
Alexandre M.O. Cukier, Patrice Therond, Svetlana A. Didichenko, Isabelle \\ Guillas-Baudouin, Samuel D. Wright, M. John Chapman, Anatol Kontush
}

\section{To cite this version:}

Alexandre M.O. Cukier, Patrice Therond, Svetlana A. Didichenko, Isabelle Guillas-Baudouin, Samuel D. Wright, et al.. Structure-function relationships in reconstituted HDL: Focus on antioxidative activity and cholesterol efflux capacity. Biochimica et Biophysica Acta Molecular and Cell Biology of Lipids, 2017, 10.1016/j.bbalip.2017.05.010 . hal-01525877

\section{HAL Id: hal-01525877 https://hal.sorbonne-universite.fr/hal-01525877}

Submitted on 22 May 2017

HAL is a multi-disciplinary open access archive for the deposit and dissemination of scientific research documents, whether they are published or not. The documents may come from teaching and research institutions in France or abroad, or from public or private research centers.
L'archive ouverte pluridisciplinaire HAL, est destinée au dépôt et à la diffusion de documents scientifiques de niveau recherche, publiés ou non, émanant des établissements d'enseignement et de recherche français ou étrangers, des laboratoires publics ou privés. 


\section{Structure-function relationships in reconstituted HDL: Focus on antioxidative activity and cholesterol efflux capacity}

Alexandre M. O. Cukier ${ }^{1}$, Patrice Therond ${ }^{2,3}$, Svetlana A. Didichenko ${ }^{4}$, Isabelle GuillasBaudouin ${ }^{1}$, M. John Chapman ${ }^{1}$, Samuel D. Wright ${ }^{4}$, Anatol Kontush ${ }^{\text {* }}$

${ }^{1}$ National Institute for Health and Medical Research (INSERM), INSERM UMR 1166 ICAN, Paris, France; University of Pierre and Marie Curie - Paris 6, Paris, France; AP-HP, Groupe Hospitalier Pitié Salpétrière, Paris, France

${ }^{2}$ AP-HP, HUPS Hôpital de Bicêtre, Le Kremlin-Bicêtre, France

${ }^{3} \mathrm{Lip}(\mathrm{Sys})^{2}$ Athérosclérose: homéostasie et trafic du cholestérol des macrophages, University Paris-Sud, University Paris-Saclay, 92296 Châtenay-Malabry. France

${ }^{4}$ CSL Behring, Bern, Switzerland and King of Prussia, USA

*Corresponding author: Dr. Anatol Kontush, INSERM UMR-S 1166 ICAN, Faculty of Medicine, Pitié-Salpêtrière 91, boulevard de l'Hôpital, 75013 Paris, France. Tel. (33) (1) 40779633. Fax (33) (1) 407796 45. E-mail anatol.kontush@upmc.fr

Running Title: Structure-function relationships in reconstituted HDL

Word count: 7899 


\section{Abstract}

Aims: High-density lipoprotein (HDL) contains multiple components that endow it with biological activities. Apolipoprotein A-I (apoA-I) and surface phospholipids contribute to these activities; however, structure-function relationships in HDL particles remain incompletely characterized.

Methods: Reconstituted HDLs (rHDLs) were prepared from apoA-I and soy phosphatidylcholine (PC) at molar ratios of 1:50, 1:100 and 1:150. Oxidative status of apoA-I was varied using controlled oxidation of Met112 residue. HDL-mediated inactivation of PC hydroperoxides (PCOOH) derived from mildly pre-oxidized low-density lipoprotein (LDL) was evaluated by HPLC with chemiluminescent detection in HDL+LDL mixtures and re-isolated LDL. Cellular cholesterol efflux was characterised in RAW264.7 macrophages.

Results: rHDL inactivated LDL-derived $\mathrm{PCOOH}$ in a dose- and time-dependent manner. The capacity of rHDL to both inactivate $\mathrm{PCOOH}$ and efflux cholesterol via ATP-binding cassette transporter A1 (ABCA1) increased with increasing apoA-I/PC ratio proportionally to the apoA-I content in $\mathrm{rHDL}$. Controlled oxidation of apoA-I Met112 gradually decreased $\mathrm{PCOOH}$-inactivating capacity of rHDL but increased ABCA1-mediated cellular cholesterol efflux.

Conclusions: Increasing apoA-I content in rHDL enhanced its antioxidative activity towards oxidized LDL and cholesterol efflux capacity via ABCA1, whereas oxidation of apoA-I Met112 decreased the antioxidative activity but increased the cholesterol efflux. These findings provide important considerations in the design of future HDL therapeutics.

Word count: 200

Keywords: apolipoprotein A-I, phosphatidylcholine, lipid hydroperoxides, oxidized LDL, Met112, ABCA1 


\section{Introduction}

Low plasma levels of high-density lipoprotein (HDL) cholesterol (HDL-C) are a cardiovascular (CV) disease risk factor [1]. This association has led to the hypothesis that raising HDL-C levels can reduce atherosclerotic disease and CV risk [2]. However, several large-scale interventional trials aimed at increasing circulating levels of HDL-C failed to decrease the incidence of CV disease in humans [3]. As a consequence of these, and also of large-scale Mendelian randomization studies, HDL-C was proposed not to be causally related to CV disease [4]. To reconcile these findings, it has been suggested that HDL does have cardioprotective activity but that HDL-C may be an inappropriate marker for such cardioprotective function [5]. Consistent with this view, measures of cellular cholesterol efflux capacity of plasma HDL show only weak correlations with HDL-C but strong correlations with coronary heart disease risk [6-8].

HDL particles contain multiple bioactive protein and lipid components that endow the lipoprotein with distinct biological activities [9]. Earlier studies have created a framework of structure-function relationships in human HDL [10-12]. Among others, biological properties of HDL include antioxidative actions towards low-density lipoprotein (LDL) and cholesterol efflux from lipid-loaded macrophages [9, 13, 14]. HDL carries several proteins which can both inhibit LDL oxidation and enhance cholesterol efflux via different mechanisms. Thus, apolipoprotein A-I (apoA-I), the major HDL protein, specifically inactivates lipid hydroperoxides (LOOH) in LDL, primary products of LDL lipid peroxidation, and effluxes cellular cholesterol via the ATP-binding cassette transporter A1 (ABCA1) [13,15]. Acting by virtue of its redox-active Met residues, Met112 and Met86, apoA-I reduces LOOH to redox-inactive lipid hydroxides, whereby Met residues become oxidized to Met sulfoxides [16-18]. This reaction involves transfer of phospholipid (PL) hydroperoxides (PLOOH) from oxidized LDL to HDL through the aqueous phase. Consistent with this notion, apoA-I is primarily reactive with $\mathrm{PLOOH}$, reflecting their surface location and direct contact with the aqueous environment $[17,18]$.

The anti-atherogenic activities of HDL can be modulated by HDL lipids, primarily by PL which predominant in the HDL lipidome [19]. Thus, sphingomyelin can decrease transfer of PLOOH from LDL to HDL, acting in part via decreasing fluidity of surface HDL lipids [18], whereas phosphatidylserine enhances both HDL-mediated protection of LDL from oxidative stress and cellular cholesterol efflux through modulating HDL surface charge [20]. Importantly, anti-atherogenic activities of HDL can be decreased in several pathologic conditions associated with elevated CV risk, such as metabolic syndrome and Type 2 diabetes [21, 22]. Such functional deficiency of HDL is paralleled by profound compositional alterations, primarily involving decreased HDL content and/or enhanced oxidation of apoA-I [23-25].

Small disc-shaped HDL particles that resemble nascent HDL can be readily generated in vitro from its major protein component, apoA-I, and phosphatidylcholine (PC). The ease of production and relatively uniform nature of these particles have allowed us to examine structure-function relationships of HDL. In particular, we have studied the impact of the ratio of protein to lipid and the extent of methionine oxidation to both $\mathrm{PC}$ hydroperoxide $(\mathrm{PCOOH})$-inactivating activity and cellular cholesterol efflux capacity of reconstituted HDL. We found that increasing relative apoA-I content in reconstituted HDL enhanced its capacity to both inactivate $\mathrm{PCOOH}$ derived from mildly oxidized LDL and efflux cholesterol from lipid-loaded macrophages via ABCA1. By contrast, oxidation of Met112 of apoA-I decreased the PCOOH inactivation but enhanced the cholesterol efflux.

Non-standard abbreviations and acronyms: AAPH, 2,2'-azobis(-amidinopropane) dihydrochloride; ABCA1, ATP-binding cassette transporter A1; apoA-I, apolipoprotein A-I; BHT, butylated hydroxytoluene; CV, cardiovascular; EDTA, ethylenediaminetetraacetic acid; HDL-C, high-density lipoprotein cholesterol; $\mathrm{LOOH}$, lipid hydroperoxides; Met(O), methionine sulfoxide; Met(112), methionine 112 residue; Met(86), methionine 86 residue; oxLDL, oxidized low-density lipoprotein; PBS, phosphate-buffered saline; PC, phosphatidylcholine; PL, phospholipid; PCOOH, phosphatidylcholine hydroperoxides; PLOOH, phospholipid hydroperoxides 


\section{Materials and Methods}

\subsection{Reconstituted HDL}

Reconstituted HDL were prepared from apoA-I isolated from human pooled plasma (CSL Behring) [26, 27] and soybean PC (Phospholipon 90, Lipoid AG/Phospholipid GmbH) by the sodium cholate dialysis method as described elsewhere [28, 29]. Reconstituted HDL particles of different size were produced using apoA-I/PL molar ratios of 1:150, 1:100, and 1:50. The conditions for reconstitution were chosen to achieve complete apoA-I and PC incorporation into lipoprotein particles (each formulation contained two apoA-I molecules per particle). After reconstitution of the particles, cholate was removed by dialysis and filtration using Biomax cassettes (NMWR $10 \mathrm{kDa}$; Millipore). Reconstituted $\mathrm{HDL}$ was stored at $-70^{\circ} \mathrm{C}$ in small aliquots or was lyophilized, sealed under vacuum and stored at $4^{\circ} \mathrm{C}$. No difference between $\mathrm{PCOOH}-$ inactivating capacity of frozen vs. lyophilised reconstituted HDL formulations was observed (Supplemental Fig. I).

Reconstituted HDL containing apoA-I with different levels of oxidation of Met112 residue were prepared using autoxidation of reconstituted $\mathrm{HDL}(1: 50 \mathrm{PC})$ at $4^{\circ} \mathrm{C}$ in the dark for various time periods. Met112 is known to be markedly more sensitive to autoxidation as compared to other Met residues of apoA-I [17]. In order to stop oxidation, vials were frozen at $-70^{\circ} \mathrm{C}$. After 5 , 14 , and 31 days of storage, levels of apoA-I oxidized at single Met 112 residue were $42 \%, 64 \%$, and $85 \%$, respectively, as determined by reverse phase HPLC method described elsewhere [16]. LC/MS/MS analysis of reconstituted HDL samples corresponding to the time points of 0,5 and 31 days of incubation at $4^{\circ} \mathrm{C}$ was performed as described by Brock et al [30]. Met112 was confirmed to be the primary site of oxidation, whereas other Met residues, Met148 and 86, were oxidized to a lesser degree (Table I, Supplemental). No oxidative modification on aromatic amino acid residues (Phe, Thr and Tyr) of apoA-I followed by protein crosslinking into dimers or higher molecular weight aggregates was detected by LS/MS/MS and SDS PAGE analysis (Supplemental Fig. II, A). In parallel, small amounts of lipid-free apoA-I were released from rHDL following extensive oxidation of Met112, while the rHDL size was only minimally modified (Supplemental Fig. II, B). Content of $\mathrm{PCOOH}$ in all reconstituted HDL formulations was low and did not exceed $0.114 \mathrm{~mol} / \mathrm{mol}$ HDL (Table II, Supplemental). Levels of oxidation of apoA-I Met112 were also low and did not exceed 6\% (Table I, Supplemental).

\subsection{Blood samples}

Blood samples were withdrawn from the cubital vein of fasting healthy normolipidemic male subjects receiving no medication. EDTA plasma (final EDTA concentration, $1 \mathrm{mg} / \mathrm{ml}$ ) was prepared from venous blood collected into sterile, evacuated tubes (Vacutainer). Plasma was immediately separated by low-speed centrifugation at $4^{\circ} \mathrm{C}$, mixed with sucrose (final concentration, $0.6 \%$ ) as a cryoprotectant for lipoproteins [31], aliquoted and frozen at $-80^{\circ} \mathrm{C}$ under nitrogen; each aliquot was thawed only once directly before analyses.

\subsection{Isolation of lipoproteins and apoA-I}

Lipoproteins were isolated from EDTA plasma or from incubation mixtures by single step, isopycnic non-denaturing density gradient ultracentrifugation in a Beckman SW41 Ti rotor at $40,000 \mathrm{rpm}$ for 44 hours in a Beckman XL70 ultracentrifuge at $15^{\circ} \mathrm{C}$ by a slight modification of the method of Chapman et al. [32] as previously described [33].

After centrifugation, each gradient was fractionated into 11 fractions corresponding to very low-density lipoprotein (VLDL) + intermediate density lipoprotein (IDL) $(\mathrm{d}<1.019 \mathrm{~g} / \mathrm{ml}), \mathrm{LDL}$ (5 subfractions, LDL1, d 1.019-1.023 g/ml; LDL2, d 1.023-1.029 g/ml ; LDL3, d 1.029-1.039 g/ml; 
LDL4, d 1.039-1.050 g/ml, and LDL5, d 1.050-1.063 g/ml) and HDL. Five major HDL subclasses were isolated, i.e., large, light HDL2b (d 1.063-1.087 g/ml) and HDL2a (d 1.088-1.110 g/ml), and small, dense HDL3a (d 1.110-1.129 g/ml), HDL3b (d 1.129-1.154 g/ml) and HDL3c (d $1.154-1.170 \mathrm{~g} / \mathrm{ml}$ ). The validity and reproducibility of this density gradient procedure, which facilitates preparative fractionation of HDL particle subspecies in a non-denaturated, native state, have been extensively documented [32, 34]. Lipoproteins were extensively dialysed against phosphate-buffered saline (PBS; $\mathrm{pH} 7.4$ ) at $4^{\circ} \mathrm{C}$ in the dark, stored at $4^{\circ} \mathrm{C}$ and used within 10 days.

Total LDL fraction was prepared by mixing LDL1, LDL2, LDL3, LDL4 and LDL5 subfractions at their equivalent plasma concentrations. Total HDL fraction was prepared by mixing HDL2b, 2a, 3a, 3b and 3c subfractions at their equivalent plasma concentrations.

Lipid-free apoA-I was isolated from human plasma as described elsewhere [35].

\subsection{Chemical analysis of lipoproteins}

Total protein, total cholesterol, free cholesterol, PL and triglyceride contents of isolated lipoprotein subfractions were determined using commercially available assays [32, 36].

Cholesteryl ester was calculated by multiplying the difference between total and free cholesterol concentrations by 1.67 [32]. Total lipoprotein mass was calculated as the sum of total protein, cholesteryl ester, free cholesterol, PL and triglyceride and expressed as plasma concentrations (mg/dl). ApoA-I content in HDL was quantitated using a commercially available kit (Diasys, France).

\subsection{PCOOH inactivating capacity of HDL and apoA-I Met oxidation}

Reference LDL obtained from a healthy normolipidemic subject was pre-oxidized at 40 $\mathrm{mg}$ total cholesterol/dl with $4 \mathrm{mM}$ of 2,2'-azobis(-amidinopropane) dihydrochloride (AAPH) for $6 \mathrm{~h}$ at $37^{\circ} \mathrm{C}$. Oxidative modification was terminated by addition of EDTA $(100 \mu \mathrm{M})$ and butylated hydroxytoluene $(\mathrm{BHT})(100 \mu \mathrm{M})$. Such short oxidation resulted in the accumulation of low amounts of $\mathrm{PCOOH}$ (between 5 and $10 \mathrm{~mol} / \mathrm{mol}$ LDL equivalent to $1-2 \%$ of total LDL PC [37]) and did not cause considerable degradation of LDL as documented by both agarose and acrylamide gel electrophoresis. Agarose gel electrophoresis was performed at the agarose concentration of $1 \%$ in $100 \mathrm{mM}$ Tris-Tricine buffer ( $\mathrm{pH} \mathrm{8.6)} \mathrm{for} 4 \mathrm{~h}$ at $60 \mathrm{~V}$. To run native $6 \%$ acrylamide gel electrophoresis, $20 \mu \mathrm{l}$ of the reaction mixture equivalent to $0.2 \mu \mathrm{g}$ protein were loaded onto the gel which was run for $10 \mathrm{~h}$ at $150 \mathrm{~V}$. All the gels were stained by Coomassie blue. The agarose gel electrophoresis revealed that relative electrophoretic mobility (REM) of LDL at the end of the oxidation equalled $1.47 \pm 0.02(n=4)$, a value well below the threshold of 3 required for the recognition of oxidized apoB by scavenger receptors [38]. Furthermore, no difference in the electrophoretic mobility of native and oxidized LDL was observed using acrylamide gel electrophoresis, indicative of the absence of the effect of the oxidation on LDL size (data not shown). Finally, agarose gel electrophoresis revealed that addition of EDTA (100 $\mu \mathrm{M})$ and butylated hydroxytoluene $(\mathrm{BHT})(100 \mu \mathrm{M})$ at the end of the oxidation fully protected LDL from further oxidative modification as documented by measurements of LDL REM (data not shown).

Oxidized LDL was dialyzed against PBS at $4^{\circ} \mathrm{C}$ to remove EDTA and excess BHT, and incubated at a concentration of $20 \mathrm{mg}$ total cholesterol/dl in the presence or absence of different concentrations of reconstituted HDL in PBS at $37^{\circ} \mathrm{C}$. Since both protein and lipid moieties of HDL are known to determine antioxidative properties of the lipoprotein [18, 20], HDL particles were compared on the basis of their total mass concentrations. EDTA $(100 \mu \mathrm{M})$ was present to inhibit lipid peroxidation during the incubation. $\mathrm{PCOOH}$ were quantified in the reaction mixture before and after incubation by HPLC with chemiluminescent detection as described elsewhere [18]. 
$\mathrm{PCOOH}$ inactivation by $\mathrm{rHDL}$ was defined as $\mathrm{PCOOH}$ decrease in oxLDL samples in the presence of rHDL relative to oxLDL alone.

To measure levels of methionine oxidation, reconstituted HDL was subjected to reverse phase HPLC using a C18 column with UV detection at $214 \mathrm{~nm}$ as described elsewhere [18]. In brief, this method allows separating and quantifying individual molecular species of oxidized apoA-I with a site-specific oxidation of Met112, Met86, or both [16]. HDL content of non-oxidized apoA-I was assessed in parallel using the same approach. No formation of oxidized species during the sample processing was observed (less than $2 \%$ oxidation).

In some experiments, total LDL and total HDL were reisolated from the reaction mixture using density gradient ultracentrifugation as described above and their content of PCOOH was evaluated. In parallel, the content of native and oxidized apoA-I Met residues in reisolated HDL was quantified by HPLC.

Molecular species of PCOOH separated by HPLC were characterised by LC/MS/MS as described elsewhere [20]. Four major species of $\mathrm{PCOOH}$ measured using chemiluminiscent detection were identified as, in the order of decreasing abundance, 18:2/16:0, 18:2/18:0, 22:6+20:4/16:0 and 22:6+20:4/18:0 PCOOH.

\subsection{Cholesterol efflux capacity of HDL}

The capacity of the HDL to efflux cellular cholesterol was assessed using $[3 \mathrm{H}]$ cholesterol-loaded RAW264.7 macrophages [35]. Before initiation of efflux, RAW264.7 cells were loaded with free $\left[{ }^{3} \mathrm{H}\right.$ ]cholesterol for $36 \mathrm{~h}$, and stimulated with $0.3 \mathrm{mM} 8 \mathrm{Br}$-cAMP for $16 \mathrm{~h}$ to up-regulate ABCA1. Efflux was initiated by incubating the $\left[{ }^{3} \mathrm{H}\right]$ cholesterol-labeled RAW264.7 cells with individual acceptors for $6 \mathrm{~h}$. Since cholesterol efflux from lipid-loaded macrophages is predominantly determined by the protein moiety of HDL [15], HDL particles were compared on the basis of their total protein concentrations. The difference in efflux between stimulated and non-stimulated cells was taken as a measure of ABCA1-mediated efflux. Kinetic parameters of ABCA1-dependent and independent cholesterol efflux mediated by rHDL were determined using the Graph Pad Prism 6 software as described elsewhere [39].

\subsection{Statistics}

Data are presented as means \pm standard deviation (SD) unless otherwise indicated. All results were analyzed for statistical significance using one-way ANOVA followed by Dunnet post-hoc test, or using t-test when appropriate. Statistical significance was set at $p<0.05$. 


\section{Results}

Reconstituted HDL with an apoA-I/PL ratio of 1:50 efficiently inactivated PC hydroperoxides in pre-oxidized LDL ( $20 \mathrm{mg}$ total cholesterol/dl equivalent to $0.24 \mu \mathrm{M}$ ) upon their co-incubation at a near-physiologic concentration ratio of $12 \mathrm{~mol} / \mathrm{mol}$ in vitro at $37^{\circ} \mathrm{C}$ (Fig. 1); in parallel, accumulation of corresponding PC hydroxides was observed (data not shown). PCOOH content of oxidized LDL (oxLDL) incubated alone slowly decreased over time, reflecting PCOOH instability (Fig. 1A); such decrease was however significantly more pronounced in the presence of reconstituted $\mathrm{HDL}$. The antioxidative effect of reconstituted $\mathrm{HDL}$ was similar for all four PCOOH species assessed, notably 18:2/16:0, 18:2/18:0, 22:6+20:4/16:0 and 22:6+20:4/18:0 $\mathrm{PCOOH}$ (data not shown).

The $\mathrm{PCOOH}$ inactivation by reconstituted $\mathrm{HDL}$ was time-dependent and increased from $12 \%$ after $0.5 \mathrm{~h}$ to $69 \%$ after $24 \mathrm{~h}$ of the incubation (Fig. 1B). The antioxidative effect of reconstituted $\mathrm{HDL}$ levelled off at 15 to $25 \%$ between 2 and $6 \mathrm{~h}$; subsequent decrease in $\mathrm{PCOOH}$ levels after a prolonged incubation of $24 \mathrm{~h}$ was associated with alterations in reconstituted $\mathrm{HDL}$ size and structure and was therefore considered physiologically of a limited importance (data not shown). As a result, incubation time of $4 \mathrm{~h}$ was selected for subsequent experiments.

A

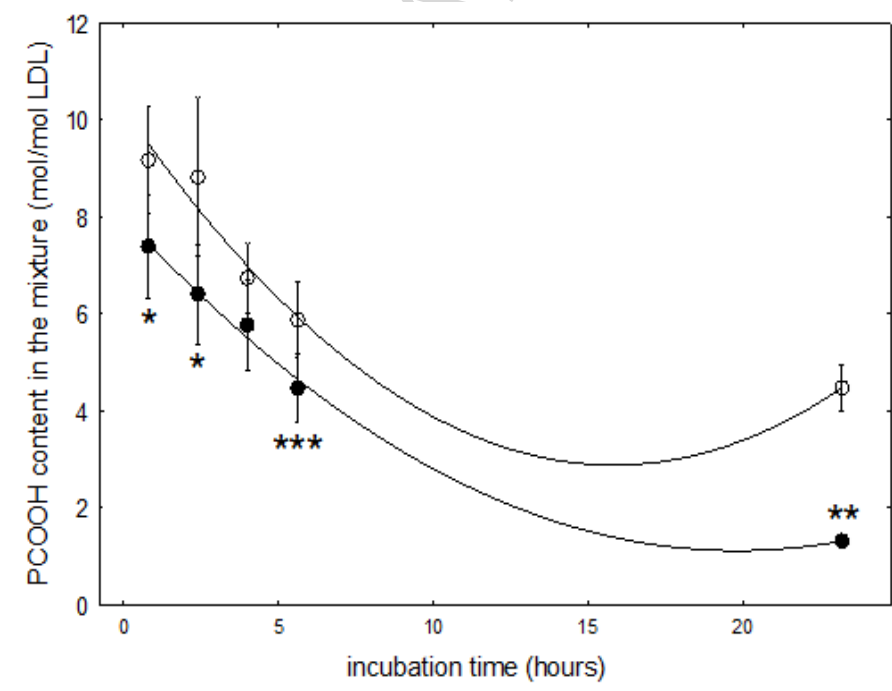

B

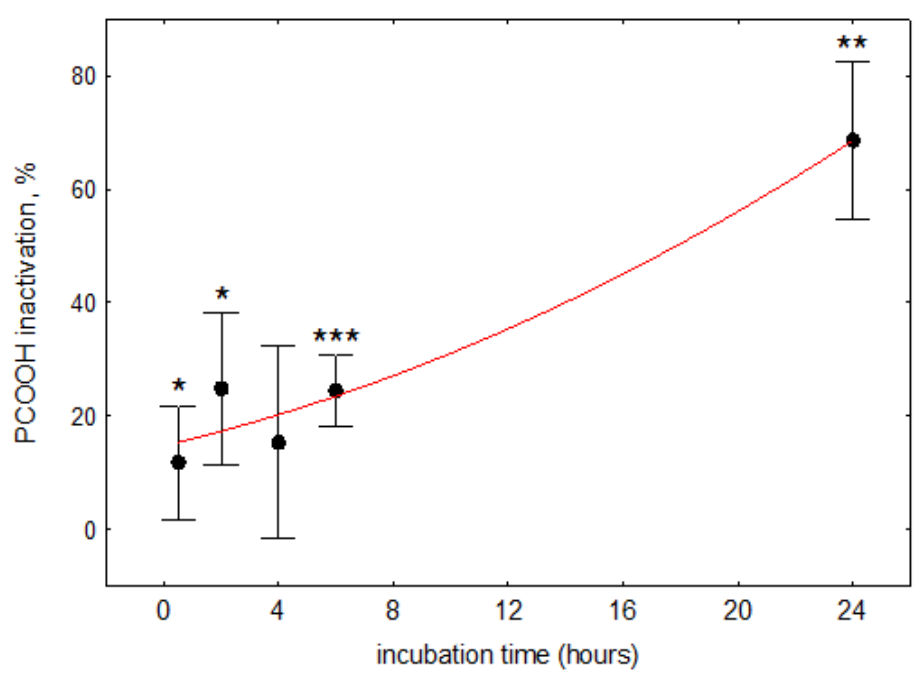


Figure 1. Time-course of the inactivation of oxLDL-derived PCOOH by reconstituted HDL (1:50 PC). (A) Evolution of $\mathrm{PCOOH}$ content in the reaction mixtures as a function of incubation time. (B) $\mathrm{PCOOH}$ inactivation in oxLDL by reconstituted $\mathrm{HDL}(1: 50 \mathrm{PC})$ as a function of incubation time. Percentage of $\mathrm{PCOOH}$ inactivation by reconstituted $\mathrm{HDL}(1: 50 \mathrm{PC})$ in the reaction mixtures relative to $\mathrm{PCOOH}$ content of oxLDL incubated alone in parallel is shown for each time point. Reconstituted HDL (1:50 PC) (40 mg mass $/ \mathrm{dl}$ ) was incubated for indicated time periods at $37^{\circ} \mathrm{C}$ in the presence of oxLDL (20 mg total cholesterol/dl) pre-oxidized by AAPH. Data of 5 independent experiments are shown as means \pm S.E.M.; ${ }^{* * *} p<0.001,{ }^{* *} p<0.01,{ }^{*} p<0.05$ vs. corresponding oxLDL sample incubated alone. The curves were plotted using polynomial fitting.

The effect of reconstituted HDL on oxLDL-derived PCOOH was dose-dependent (Fig. 2). No hydroperoxide inactivation was detected when the reconstituted $\mathrm{HDL}$ was present at a low concentration of $10 \mathrm{mg}$ mass/dl (Fig. 2A). The PCOOH inactivation however increased to over $20 \%$ at higher concentrations of 20 and $40 \mathrm{mg} / \mathrm{dl}$; further elevation of the reconstituted $\mathrm{HDL}$ concentration to $80 \mathrm{mg} / \mathrm{dl}$ did not enhance the antioxidative effect (Fig. 2B). The concentration of $20 \mathrm{mg} / \mathrm{dl}$ was therefore selected for subsequent experiments.
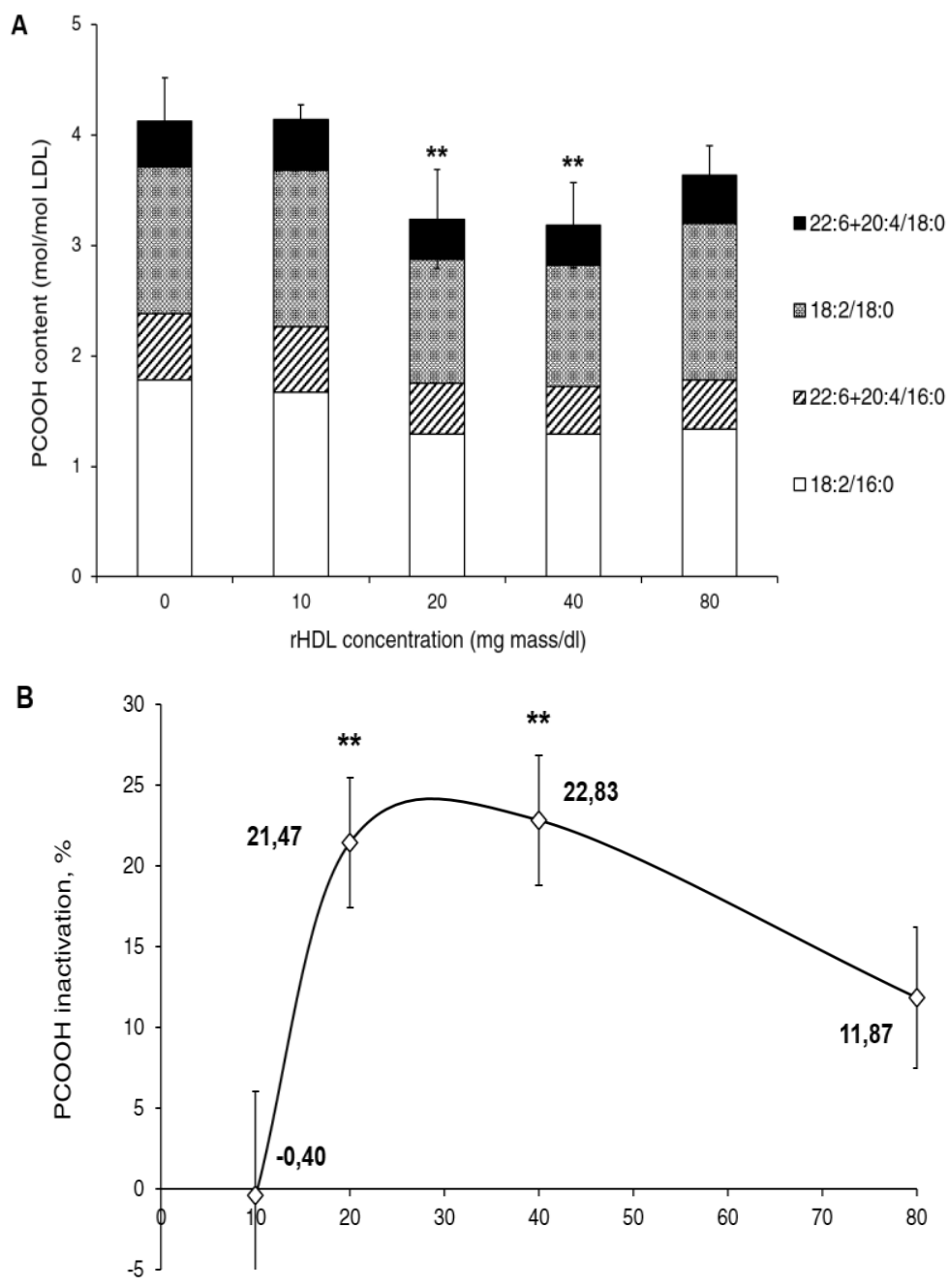

rHDL content (mg mass/dL)

Figure 2. Dose-dependence of the inactivation of oxLDL-derived PCOOH by reconstituted HDL (1:50 PC). (A) PCOOH content in oxLDL and reconstituted HDL (1:50 PC) reaction mixtures as a function of reconstituted HDL (1:50 PC) concentration. (B) PCOOH inactivation in oxLDL by reconstituted HDL 
(1:50 PC) as a function of reconstituted HDL concentration. Percentage of $\mathrm{PCOOH}$ inactivation by reconstituted $\mathrm{HDL}(1: 50 \mathrm{PC})$ in the reaction mixtures is shown for each reconstituted HDL (1:50 PC) concentration relative to PCOOH content of oxLDL incubated alone. Reconstituted HDL (1:50 PC) (0 to 80 $\mathrm{mg}$ mass $/ \mathrm{dl}$ ) was incubated for $4 \mathrm{~h}$ at $37^{\circ} \mathrm{C}$ in the presence of oxLDL (20 mg total cholesterol/dl) preoxidized by AAPH. Data of 3 independent experiments are shown as means \pm S.E.M.; ${ }^{* *} p<0.01$ vs. oxLDL incubated alone.

The capacity of reconstituted HDL to inactivate oxLDL-derived PCOOH was evaluated in several reconstituted HDL formulations differing in their chemical composition. First, three reconstituted $\mathrm{HDL}$ formulation displaying apoA-I/PC molar ratios of 1:50, 1:100 and 1:150 were compared. We found that the $\mathrm{PCOOH}$-inactivating capacity of such particles decreased with decreasing apoA-I/PC ratio (Fig. 3A). Interestingly, on an HDL mass basis, the absolute activity was markedly lower than that displayed by plasma HDL3 isolated from a healthy normolipidemic donor (Fig. 3A). Since HDL3 isolated by our density gradient ultracentrifugation approach displays both PON1 and PAF-AH activities [36], they can contribute to the enhanced capacity of HDL3 to inactivate $\mathrm{PCOOH}$ relative to $\mathrm{rHDL}$.

Replotting the data on the basis of apoA-I content rather than total mass reveals that the capacity of reconstituted HDLs to inactivate $\mathrm{PCOOH}$ was directly proportional to the apoA-I concentration in the reaction mixtures (Fig. 3B). By contrast, the relationship between $\mathrm{PCOOH}-$ inactivating capacity of rHDL and its PC content tended to inverse (Fig. 3C). Interestingly, lipidfree apoA-I isolated from human plasma did not reveal $\mathrm{PCOOH}$-inactivating activity in this assay $(6.5 \pm 3.9 \%$ of inactivated $\mathrm{PCOOH}$ at $8 \mathrm{mg}$ apoA-l/dl; $\mathrm{n}=3)$. Similarly, $\mathrm{PCOOH}$ inactivation by plasma-derived HDL was not enhanced by the plasma pre-incubation with lipid-free apoA-I (Table III Supplemental).

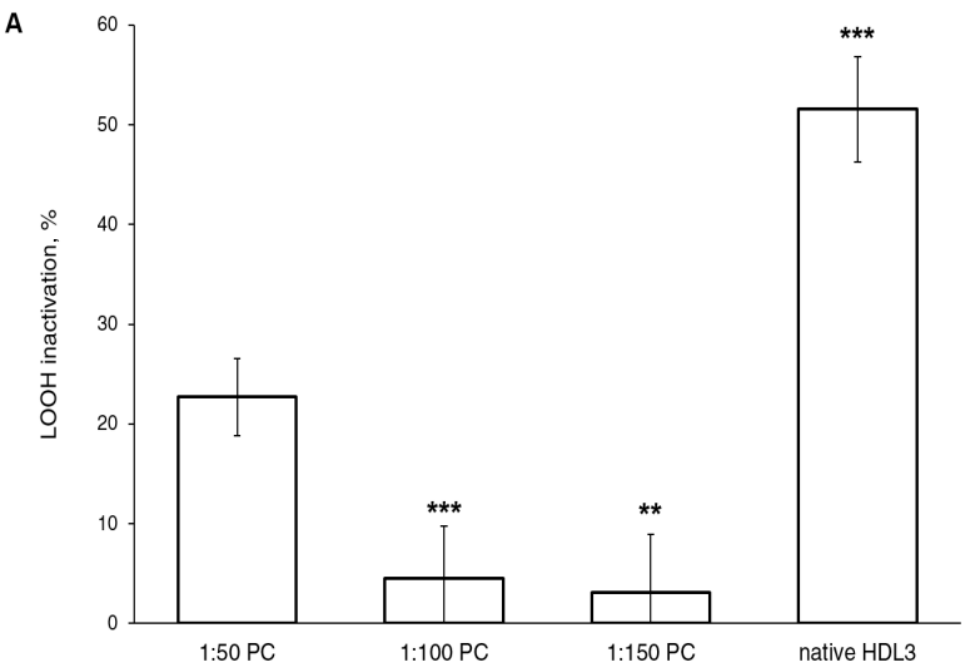




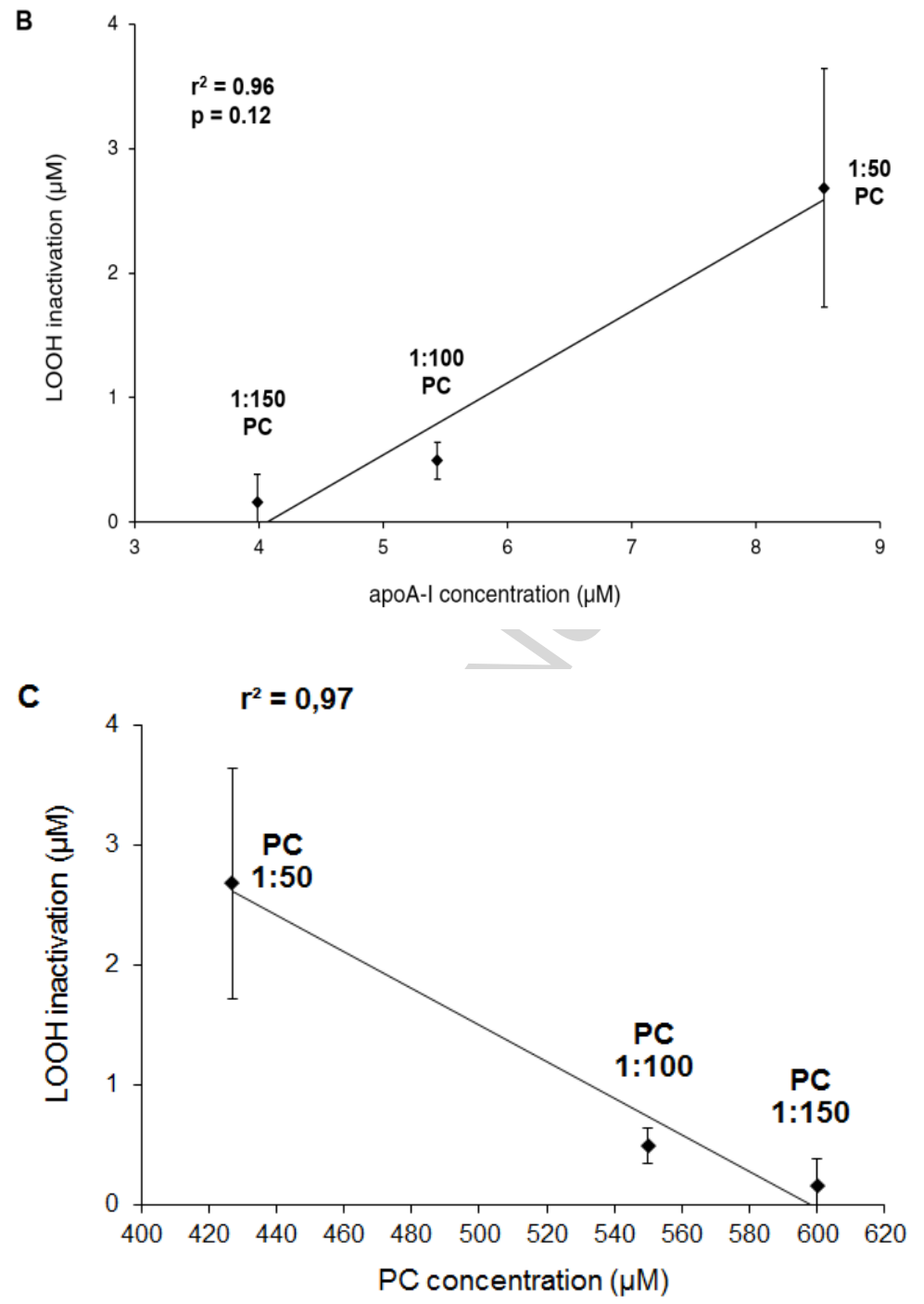

Figure 3. Inactivation of oxLDL-derived $\mathrm{PCOOH}$ by reconstituted $\mathrm{HDL}$ formulations differing in their lipid content. (A) PCOOH inactivation by reconstituted HDLs in their reaction mixtures with oxLDL. $\mathrm{PCOOH}$ inactivation by plasma HDL3 evaluated in parallel under identical conditions is shown for comparison. Percentage of $\mathrm{PCOOH}$ inactivation by $\mathrm{HDL}$ is shown relative to $\mathrm{PCOOH}$ content of oxLDL incubated alone. (B) $\mathrm{PCOOH}$ inactivated by reconstituted HDLs as a function of apoA-I concentration in the reaction mixture. (C) $\mathrm{PCOOH}$ inactivated by reconstituted $\mathrm{HDLs}$ as a function of $\mathrm{PC}$ concentration in the reaction mixture. Reconstituted HDL (20 mg mass $/ \mathrm{dl}$ ) was incubated for $4 \mathrm{~h}$ at $37^{\circ} \mathrm{C}$ in the presence of oxLDL (20 mg total cholesterol/dl) pre-oxidized by AAPH. Data of at least 3 independent experiments are shown; ${ }^{* * *} p<0.001,{ }^{* *} p<0.01$ vs. reconstituted HDL (1:50 PC).

Having established the dependence of $\mathrm{PCOOH}$ inactivation on apoA-I content, we next asked about the effect of apoA-I with oxidized methionine residues. Reconstituted HDL particles of a fixed apoA-I/PC molar ratio of 1:50 but differing in the level of oxidation of Met112 residue of apoA-I from 6 to $85 \%$ were prepared by controlled oxidation. We observed that such approach gradually decreased $\mathrm{PCOOH}$-inactivating capacity of reconstituted HDL (Fig. 4). Extensive (85\%) oxidation of Met112 in apoA-I resulted in the complete loss of the $\mathrm{PCOOH}$-inactivating capacity (Fig. 4A). As a consequence, the capacity of reconstituted HDLs containing the oxidized 
Met residue to inactivate $\mathrm{PCOOH}$ was directly proportional to the concentration of non-oxidized apoA-I in the reaction mixtures (Fig. 4B).
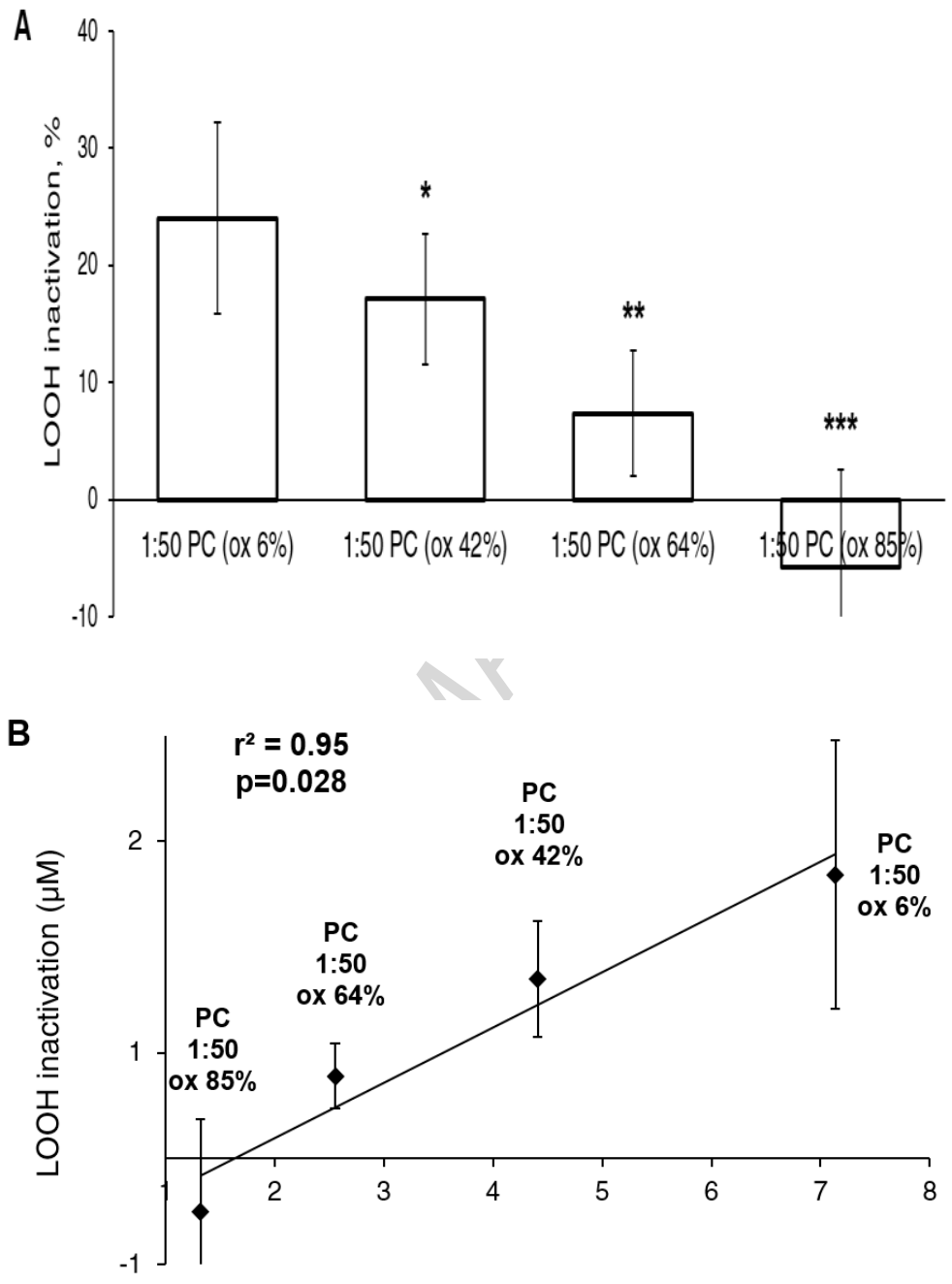

non-oxidised apoA-I concentration $(\mu \mathrm{M})$

Figure 4. Inactivation of oxLDL-derived PCOOH by reconstituted HDL (1:50 PC) formulations differing by the level of apoA-I Met112 oxidation. (A) PCOOH inactivation by reconstituted HDLs in their reaction mixtures with oxLDL. Percentage of $\mathrm{PCOOH}$ inactivation by $\mathrm{HDL}$ is shown relative to $\mathrm{PCOOH}$ content of oxLDL incubated alone. (B) PCOOH inactivated by reconstituted HDLs as a function of the concentration of non-oxidized apoA-I in the reaction mixture. Reconstituted HDL (20 mg mass $/ \mathrm{dl}$ ) was incubated for $4 \mathrm{~h}$ at $37^{\circ} \mathrm{C}$ in the presence of oxLDL (20 mg total cholesterol/dl) pre-oxidized by AAPH. The level of apoA-I Met oxidation is shown for each HDL preparation as \% of total apoA-I content. Data of 4 independent experiments are shown; ${ }^{* * *} p<0.001$, ${ }^{* *} p<0.01,{ }^{*} p<0.05$ vs. reconstituted HDL (1:50) with Met112 oxidized by $6 \%$.

In order to verify that reconstituted HDLs were capable of decreasing $\mathrm{PCOOH}$ content of pre-oxidized LDL, we re-isolated LDL from the reaction mixture following its incubation with reconstituted HDL. We observed that first, reconstituted HDL (1:50 PC) decreased PCOOH content of re-isolated LDL by 39\% (Fig. 5). This effect was similar for all molecular species of $\mathrm{PCOOH}$ (data not shown). Second, extensive oxidation of apoA-I Met112 in reconstituted HDL 
resulted in the complete loss of the capacity of reconstituted HDL to inactivate PCOOH in reisolated LDL (Fig. 5). The results obtained in re-isolated LDL were therefore similar to those obtained in reconstituted HDL and LDL mixtures (Fig. 4). The antioxidative effects measured in the reaction mixture were however consistently lower relative to those observed in re-isolated LDL (Fig. 5).

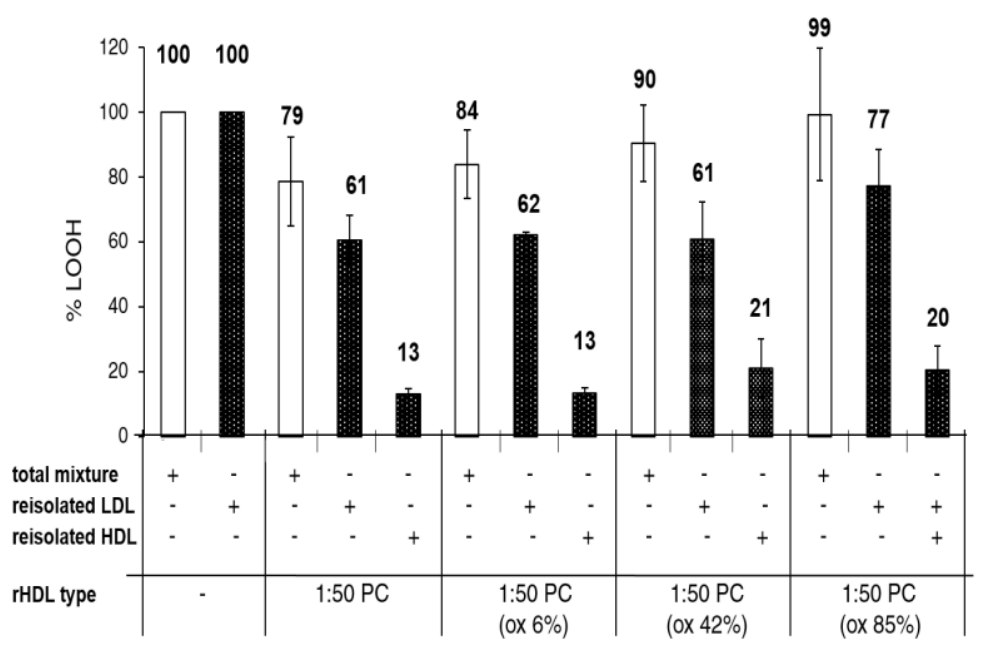

Figure 5. Inactivation of oxLDL-derived PCOOH in re-isolated LDL by reconstituted HDL (1:50 PC) differing by the level of apoA-I Met112 oxidation, and PCOOH content of re-isolated HDL.

Preoxidized LDL was incubated with HDL preparations as shown. Open bars depict the level of PCOOH in complete reaction mixtures normalized to the level seen without added HDL. PCOOH was again measured after reisolation of the LDL and HDL. Closed bars depict PCOOH in individual fractions normalized to the $\mathrm{PCOOH}$ measured in LDL reisolated after incubation without HDL. Numbers depict $\mathrm{PCOOH}$ content as a percentage relative to the corresponding initial levels. Data of 3 independent experiments are shown.

In parallel with the decrease in $\mathrm{PCOOH}$ content of LDL, $\mathrm{PCOOH}$ content of re-isolated HDL increased (Fig. 5). Initial content of PCOOH in all reconstituted HDL formulations was low (Table II, Supplemental) and did not significantly contribute to the levels measured following incubations with oxLDL. The accumulation of $\mathrm{PCOOH}$ in reconstituted $\mathrm{HDL}$ was enhanced by apoA-I Met112 oxidation (Fig. 5). The relative distribution of PCOOH between LDL and reconstituted HDL did not however depend on the level of Met112 oxidation (Supplement Fig. III). Indeed, LDL re-isolated from the reaction mixtures contained about two-thirds of the total $\mathrm{PCOOH}$ independently of the oxidation level of Met112 in reconstituted HDL, while re-isolated HDL carried the remainder.

Incubation of reconstituted HDL with oxLDL resulted in further oxidation of apoA-I Met residues (Fig. 6). The content of non-oxidized apoA-I in the reconstituted HDL (1:50 PC) decreased after the incubation, whereas that of oxidized apoA-I species increased (Fig. 6A). There was no apparent specificity in the accumulation of individual molecular species of oxidized apoA-I with a site-specific oxidation of Met112, Met86 or both (Fig. 6A). Similar results were obtained using HDL reconstituted with different amounts of $\mathrm{PC}$ at the apoA-I/PC molar ratios of 1:100 (data not shown) and 1:150 (Fig. 6B).

In these studies, we noted that low amounts of oxidized methionines accumulated in apoA-I during incubation in the absence of oxLDL. This autoxidation was lowest in native HDL ( 6\%) and highest in reconstituted HDL with the highest PL content (up to 40\%). As a consequence, the oxidation of Met residues in apoA-I upon incubation with oxLDL (i.e. difference 
between HDL content of non-oxidized apoA-I before and after the incubation) was less pronounced in rHDL with a higher lipid content (cf. Fig. 6A and 6B). Thus, Met residues of apoA-I in the apoA-1/1:50 PC and 1:150 PC particles were oxidized by $55.0 \pm 4.9$ and $41.7 \pm 5.8 \%$, respectively, during the incubation in the presence of oxLDL $(n=5)$. ApoA-I Met residues of native HDL3 were more sensitive to the exposure to oxLDL, with the total increase in oxidized Met of $71.6 \pm 0.4 \%$ of total apoA-I at the end of the incubation $(n=4)$. Interestingly, Met residues of lipidfree apoA-I were only weakly sensitive to the exposure to oxLDL, with the total content of oxidized Met increasing from $15.8(15.8-15.9)$ to $22.2(21.8-22.6) \%$ of total apoA-I at the end of the incubation $(n=2)$.

A
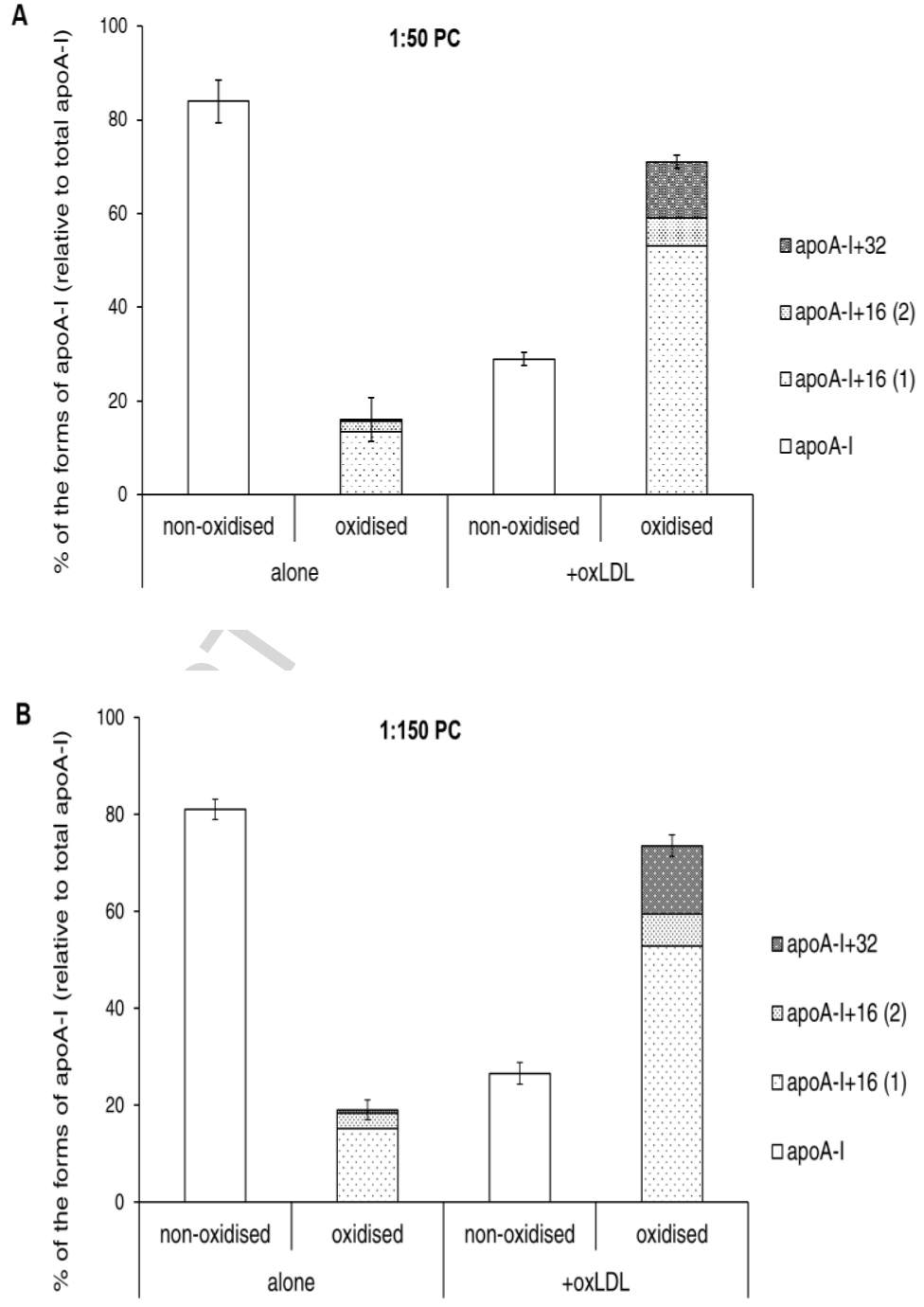

Figure 6. Oxidation of Met residues of apoA-I derived from reconstituted HDL formulations differing in their lipid content. Contents of non-oxidized and oxidized forms of apoA-I expressed as a percentage of total apoA-I are shown for reconstituted HDL of the apoA-I/PC molar ratios of 1:50 (A) and 1:150 (B). Reconstituted HDL (20 mg mass/dl) was incubated for $4 \mathrm{~h}$ at $37^{\circ} \mathrm{C}$ in the absence (alone) or in the presence of oxLDL (20 mg total cholesterol/dl) pre-oxidized by AAPH. Data of 3 independent experiments are shown. 
As expected, controlled autoxidation of reconstituted $\mathrm{HDL}$ resulted in an accumulation of apoA-I species containing oxidized Met residues and in a decrease in non-oxidized apoA-I (Fig. 7). As a consequence, oxidation of Met residues in apoA-I upon incubation with oxLDL (difference between HDL content of non-oxidized apoA-I before and after the incubation) decreased with increasing initial Met112(O) content in reconstituted HDL (Fig. 7A). Similarly, differences between HDL content of oxidized apoA-I Met before and after the incubation diminished with increasing baseline Met oxidation (Fig. 7B). As a consequence, PCOOH inactivation by reconstituted HDL formulations was proportional to consumption of apoA-I Met upon incubation with oxLDL (Fig. 7C).

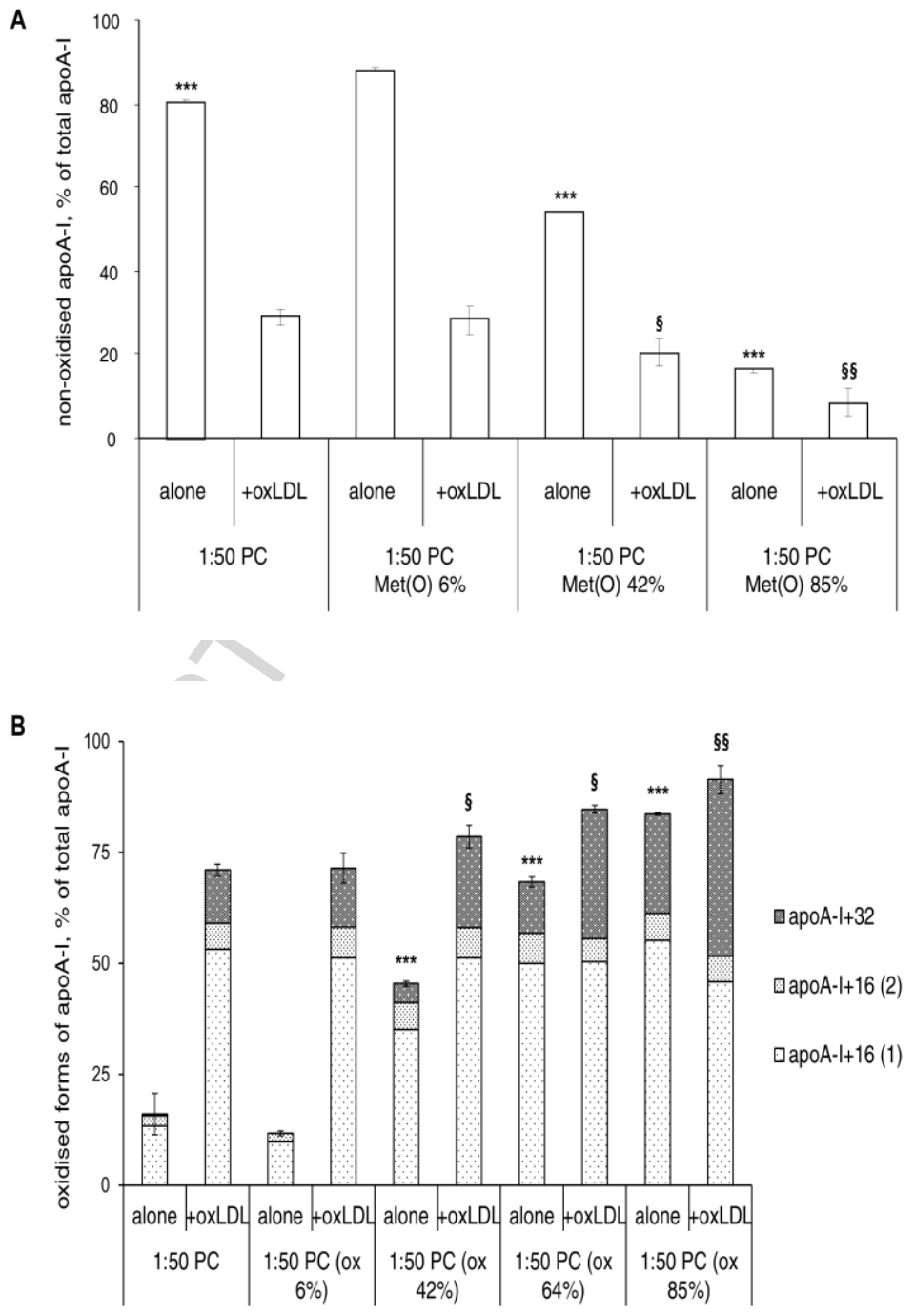


C

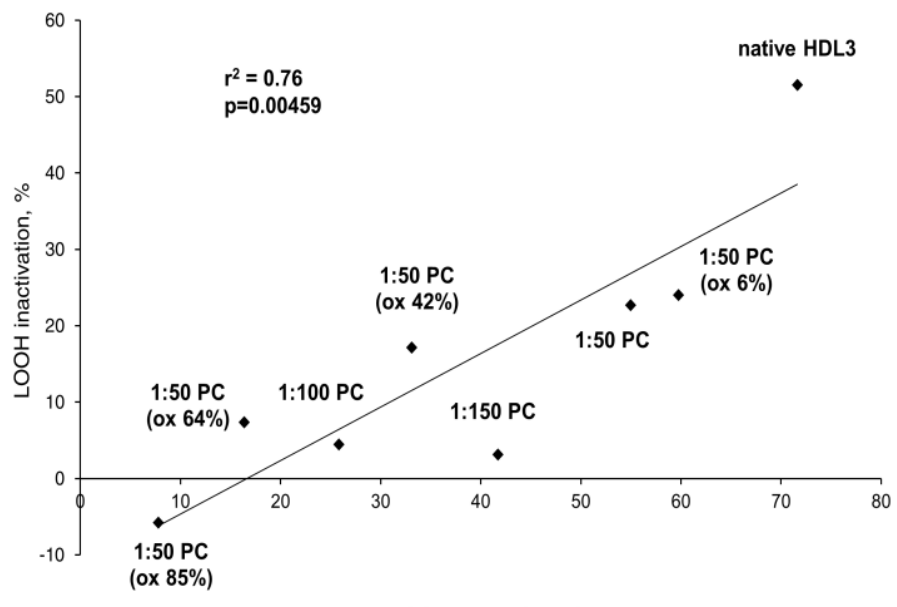

apoA-I consumption, \%

Figure 7. Oxidation of Met residues of apoA-I derived from reconstituted HDL (1:50 PC)

formulations differing by the level of apoA-I Met112 oxidation. (A) Content of non-oxidized apoA-I expressed as a percentage of total apoA-I. (B) Content of Met-oxidized species of apoA-I expressed as a percentage of total apoA-I. Reconstituted HDL (20 mg mass $/ \mathrm{dl})$ was incubated for $4 \mathrm{~h}$ at $37^{\circ} \mathrm{C}$ in the absence (alone) or in the presence of oxLDL ( $20 \mathrm{mg}$ total cholesterol/dl) pre-oxidized by AAPH. Data of 3 independent experiments are shown; ${ }^{* * *} p<0.001$ vs. HDL 1:50 PC Met(O) $1126 \%$ alone; $\S \S<0.01$, $\mathrm{p}<0.05$ vs. HDL 1:50 PC Met(O)112 6\% + oxLDL. (C) PCOOH inactivation by all studied reconstituted $\mathrm{HDL}$ formulations as a function of apoA-I consumption in the reaction mixture. Reconstituted HDL $(20 \mathrm{mg}$ mass $/ \mathrm{dl}$ ) was incubated for $4 \mathrm{~h}$ at $37^{\circ} \mathrm{C}$ in the presence of oxLDL (20 mg total cholesterol/dl) pre-oxidized by AAPH. The level of apoA-I Met oxidation is shown in brackets as \% of total apoA-I content. Data of 4 independent experiments are shown; ${ }^{* *} p<0.01$ vs. 1:50 PC Met(O)112 6\%.

We next determined the effect of reconstituted HDL with different chemical composition and different states of methionine oxidation on cellular cholesterol efflux. The capacity of reconstituted $\mathrm{HDL}$ to efflux $[3 \mathrm{H}]$-cholesterol from lipid-loaded RAW264.7 macrophages via ABCA1 increased with increasing apoA-I/PC ratio (Fig. 8A). On the other hand, ABCA1independent cholesterol efflux was inversely related to the apoA-I/PC ratio in reconstituted HDL (Fig. 8B). Consistent with these data, $V_{\max }$ values calculated for $A B C A 1-d e p e n d e n t ~ e f f l u x$ were 2.1-fold higher for HDL 1:50 relative to HDL 1:150 (Table IV Supplemental). By contrast, $V_{\max }$ values calculated for ABCA1-independent efflux were 1.7-fold lower for HDL 1:50 relative to HDL 1:150. In parallel, relative catalytic efficiency of ABCA1-dependent efflux increased while that of ABCA1-independent efflux decreased with increasing apoA-I/PC ratio (Table IV Supplemental). 

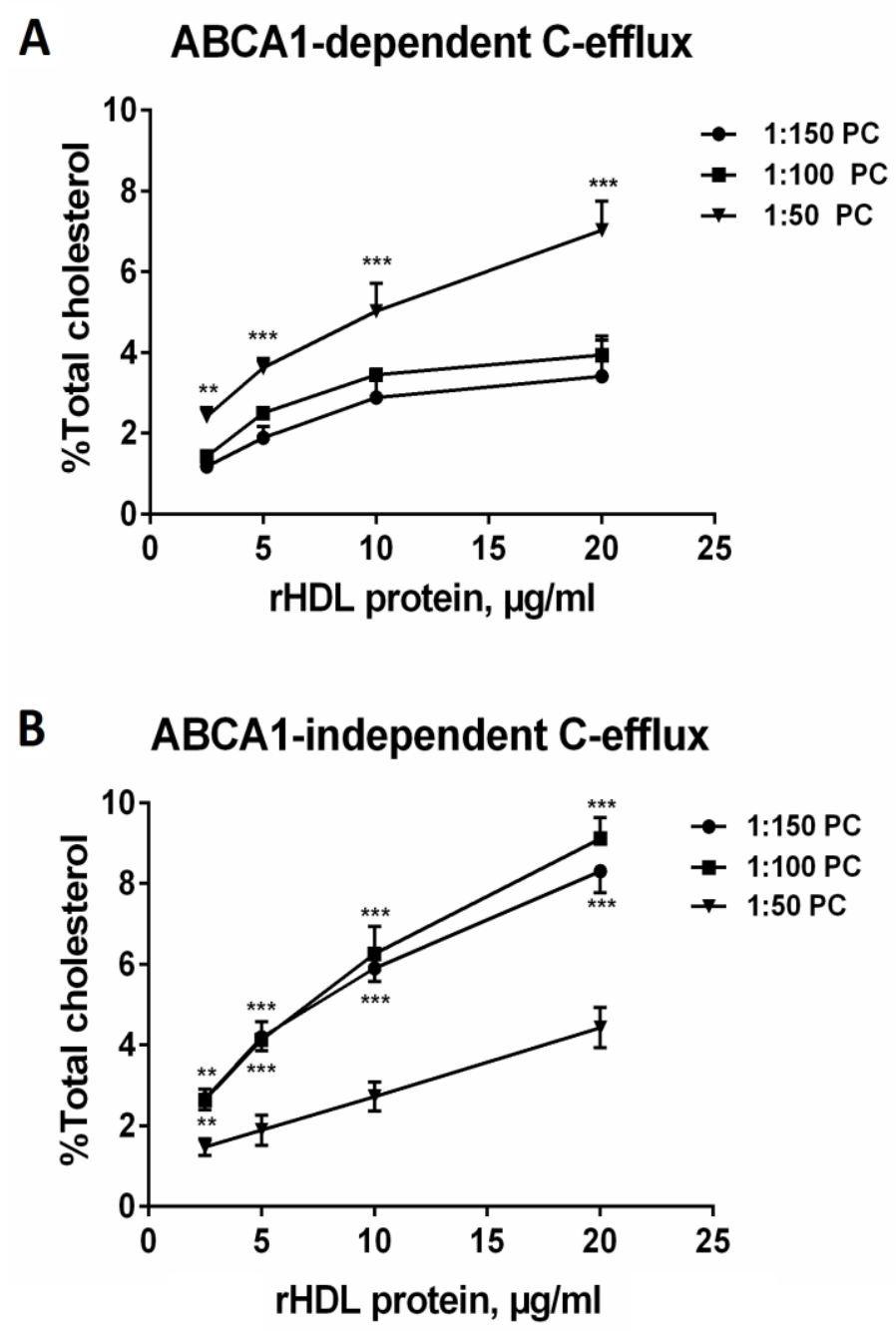

Figure 8. Cholesterol efflux from RAW264.7 lipid-loaded macrophages to reconstituted HDL formulations differing in their lipid content. $A B C A 1$-dependent $(A)$ and independent $(B)$ cholesterol efflux is shown. RAW264.7 cells were loaded with free $[3 \mathrm{H}]$ cholesterol for $36 \mathrm{~h}$ and stimulated with $0.3 \mathrm{mM}$ $8 \mathrm{Br}$-cAMP for $16 \mathrm{~h}$ to up-regulate $\mathrm{ABCA} 1$. Efflux was promoted by incubating the $[3 \mathrm{H}]$ cholesterol-labeled RAW264.7 cells with each individual acceptor for $6 \mathrm{~h}$. Difference in the efflux between stimulated and nonstimulated cells was used as a measure of ABCA1-mediated efflux. Data of at least 3 independent experiments are shown; for ABCA1-dependent efflux, ${ }^{* *} p<0.001,{ }^{* *} p<0.01$ vs. HDL (1:50 PC); for ABCA1-independent efflux, ${ }^{* *} p<0.001,{ }^{* *} p<0.01$ vs. HDL (1:150 PC).

Finally, controlled oxidation of apoA-I Met112 increased ABCA1-mediated cholesterol efflux from RAW164.7 cells but did not affect ABCA1-independent efflux (Fig. 9). 


\section{A} ABCA1-dependent C-efflux

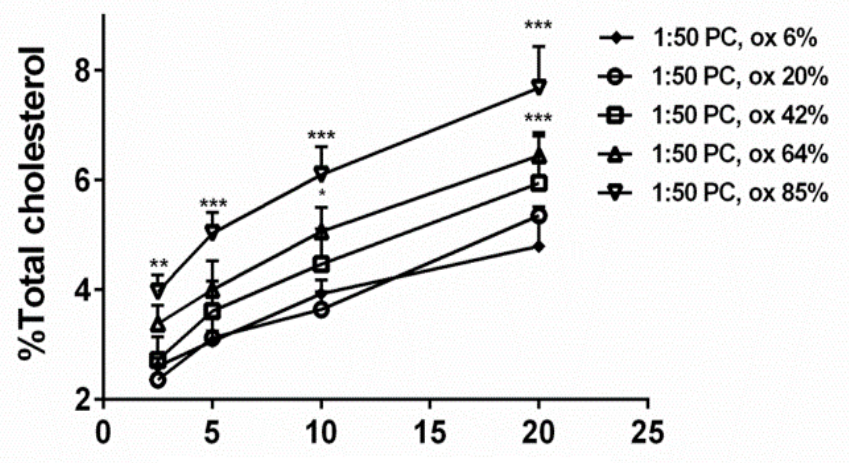

rHDL protein, $\mu \mathrm{g} / \mathrm{ml}$

B ABCA1-independent C-efflux

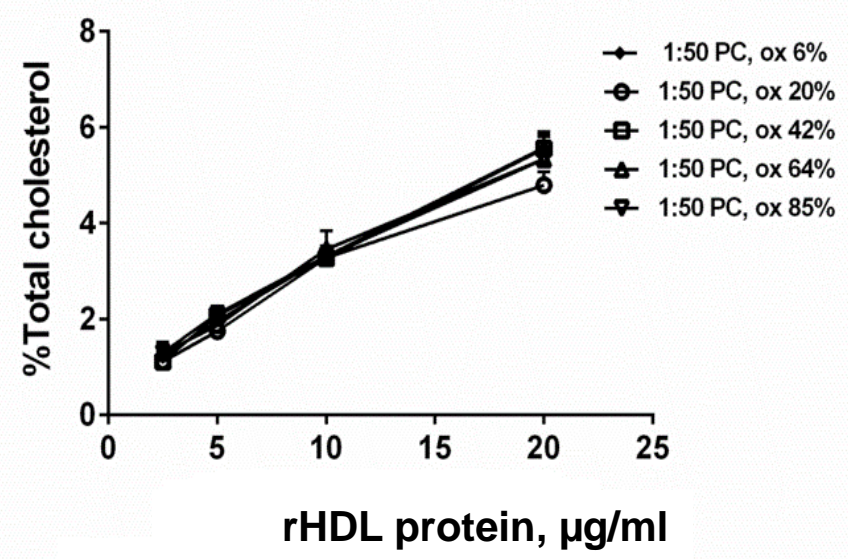

Figure 9. Cholesterol efflux from RAW264.7 lipid-loaded macrophages to reconstituted HDL (1:50 PC) formulations differing by the level of apoA-I Met112 oxidation. ABCA1-dependent (A) and independent (B) cholesterol efflux is shown. RAW264.7 cells were loaded with free $\left[{ }^{3} \mathrm{H}\right]$ cholesterol for $36 \mathrm{~h}$ and stimulated with $0.3 \mathrm{mM} 8 \mathrm{Br}-\mathrm{CAMP}$ for $16 \mathrm{~h}$ to up-regulate ABCA1. Efflux was promoted by incubating the $\left[{ }^{3} \mathrm{H}\right]$ cholesterol-labeled RAW264.7 cells with each individual acceptor for $6 \mathrm{~h}$. Difference in the efflux between stimulated and non-stimulated cells was used as a measure of ABCA1-mediated efflux. Data of at least 3 independent experiments are shown; ${ }^{* *} p<0.001,{ }^{* *} p<0.01$ vs. HDL (1:50 PC ox 6\%). 


\section{Discussion}

Our studies of structure-function relationships in reconstituted HDL particles focussed on the antioxidative activity and cellular cholesterol efflux capacity of HDL, both known to belong to the major anti-atherogenic properties of the lipoprotein $[9,13,14]$. We evaluated inactivation of $\mathrm{PCOOH}$ in mildly oxLDL and efflux of cholesterol from lipid-loaded macrophages as two metrics of HDL function. In terms of the structure and composition of HDL, we studied reconstituted HDL containing only apoA-I and PC as two essential components and varied the apoA-I content of the particle relative to $P L$ as well as the level of apoA-I oxidation. Our data revealed that the capacity to both inactivate oxLDL-derived $\mathrm{PCOOH}$ and efflux cellular cholesterol via ABCA1 increased with increasing apoA-I content in reconstituted HDL. In contrast, controlled oxidation of Met112 residue of apoA-I decreased $\mathrm{PCOOH}$ inactivation but increased cholesterol efflux.

$\mathrm{HDL}$ is well established to protect LDL from oxidative stress induced by one-electron oxidants, such as free radicals $[13,40,41]$, resulting in the inhibition of the production of proinflammatory oxidized lipids, including LOOH and short-chain oxidized PLs. On the other hand, HDL is unlikely to protect LDL from oxidation induced by two-electron oxidants, such as hypochlorite and peroxynitrite, which directly attack proteins and only weakly modify lipid moieties [42]. Mechanisms of the HDL-mediated inactivation of LOOH involve initial transfer of PLOOH from oxLDL to HDL with subsequent reduction of PLOOH by redox-active Met residues of apoA-I, which results in the formation of PL hydroxides (PLOH) and methionine sulfoxides [9, 36]. By contrast, hydrolytic inactivation of short-chain oxidized PL by HDL is primarily derived from the action of HDL-associated enzymes, such as paraoxonase 1 and platelet activating factor acetyl hydrolase (PAF-AH) [13,40]. In addition to the inactivation of lipid hydroperoxides, apoA-I equally provides a key contribution to the capacity of HDL to efflux cholesterol from lipidloaded macrophages [15]. This process is predominantly mediated by ABCA1 which is overexpressed on the cell surface upon lipid loading [43].

HDL particles are however highly heterogeneous in their composition, metabolism and function [44]. Indeed, small, dense, protein-rich HDL3 provide more efficient protection of LDL from oxidative stress and display higher cholesterol efflux capacity from lipid-loaded macrophages as compared to large, light, lipid-rich HDL2 [20]. Native HDLs are however characterised by a complex composition, rendering a clear-cut conclusion on the links between the function and protein-to-lipid ratio in HDL not straightforward. It is important in this regard that our present structure-function analysis using model HDL particles of simplified composition confirms the direct relationship of the protein-to-lipid ratio with two major metrics of HDL function, and notably with the antioxidative and cholesterol effluxing activities. As a corollary, the both metrics were directly associated with HDL density and inversely associated with HDL size, as already observed in native plasma HDL [20].

The increase in the anti-atherogenic activities of HDL at higher protein-to-lipid ratios can be most straightforwardly accounted for by the elevated particle content of apoA-I, the major functional $\mathrm{HDL}$ component. This conclusion is consistent with data on $\mathrm{PCOOH}$ inactivation obtained at a fixed total HDL mass. In a similar fashion, our earlier data revealed that reconstituted HDL particles displaying elevated protein-to-lipid ratios showed enhanced capacity to efflux cholesterol from macrophages when compared on a total mass basis [15]. By contrast, our present data demonstrate that protein-rich reconstituted HDL containing only apoA-I and PC was a more efficient acceptor of cellular cholesterol relative to their lipid-rich counterparts when compared on a total protein basis. These data indicate that, other than apoA-I content, HDL characteristics contribute to the potent cholesterol-effluxing properties of small, lipid-rich particles. Data on the immunoreactivity of HDL subpopulations towards a set of anti-apoA-I antibodies recognizing distinct epitopes of the protein suggest that apoA-I conformation can be modulated by HDL lipid content, resulting in enhanced interaction with ABCA1 of lipid-poor subspecies [15, 45]. As a consequence, differential conformational modulation of apoA-I might result in an 
improved accessibility of redox-active Met residues for the interaction with oxLDL-derived $\mathrm{PCOOH}$ molecules and hence enhanced $\mathrm{PCOOH}$ inactivation in small, protein-rich HDL.

Interestingly, lipid-free apoA-I isolated from human plasma revealed only a weak capacity to inactivate oxLDL-derived $\mathrm{PCOOH}$, indicating that some lipidation is required for the antioxidative activity of the protein. This finding may reflect the essential role of the surface PL monolayer for the efficient transfer of $\mathrm{PLOOH}$ to apoA-I/lipid complexes with subsequent redox inactivation.

Consistent with the key role of redox-active Met residues for the lipid hydroperoxideinactivating capacity of HDL, gradual oxidation of Met112 resulted in a decrease in this activity with complete loss at an elevated degree of oxidation. Interestingly, such oxidation differentially affected HDL-mediated cholesterol efflux from macrophages, which increased in parallel with the accumulation of Met112(O). This observation can be accounted for by elevated conformational lability of apoA-I upon mild HDL oxidation [46, 47], similar to that employed in our study to progressively oxidize Met112. In addition, apoA-I association with lipids is attenuated by mild oxidation secondary to the addition of oxygen atoms to the protein structure $[40,48]$. Consistent with these findings, we detected small amounts of lipid-free apoA-I in rHDL formulations with a high content of Met112(O) (Supplemental Fig. II). Importantly, lipid-free apoA-I represents an excellent acceptor of cellular cholesterol via ABCA1 [15]. Acting together, elevated conformational lability and diminished lipid affinity of mildly oxidized apoA-I can be expected to enhance release of the protein from HDL and its exchange between HDL particles and the ABCA1 transporter, facilitating cholesterol efflux from cells.

Reconstituted HDL formulations are currently in clinical trials for the treatment of cardiovascular disease [5]. We have previously shown that one such formulation, CSL112 [49], elevated plasma cholesterol efflux capacity in both healthy volunteers [50] and patients with atherothrombotic disease [51]. The results presented here suggest that CSL112 may also enhance antioxidative activity of the plasma HDL pool, providing another potential benefit. In recent in vivo studies, we found that parent CSL112 particles were extensively remodelled post infusion, yielding both larger and smaller daughter species [52]. Studies of purified products of such remodeling revealed that species of small and intermediate size displayed both strong cholesterol efflux capacity and antioxidative activity, while lipid-poor apoA-I possessed strong cholesterol efflux capacity but weak antioxidative activity [52]. These findings are fully consistent with our present data, emphasizing both the broad spectrum of structural forms in which apoA-I may circulate and the critical role of their structure in determining HDL function.

Our studies were not free of limitations, which primarily involved use of HDL particles in the absence of other relevant components of plasma, especially albumin, which can modulate the effects of rHDL. Despite this, our structure-function analysis of reconstituted HDL highlights important considerations for the design of future HDL therapeutics. Available data suggest that deficient HDL-mediated protection of LDL from oxidative stress and defective cellular cholesterol efflux capacity can both contribute to accelerated atherogenesis [53, 54]. As a corollary, normalization of the atheroprotective properties of HDL possesses the potential to diminish oxidative modification of LDL in the arterial intima and to accelerate cholesterol removal from macrophages, thereby inducing plaque stabilization and/or regression and attenuating disease development. Small, dense reconstituted HDL particles enriched in apoA-I relative to PC represent a primary therapeutic option to be developed in this regard.

\section{Grant support}

These studies were funded by the award of a Research Grant from CSL Behring to Drs M.J. Chapman and A. Kontush. 


\section{Disclosures}

None relevant to this study.

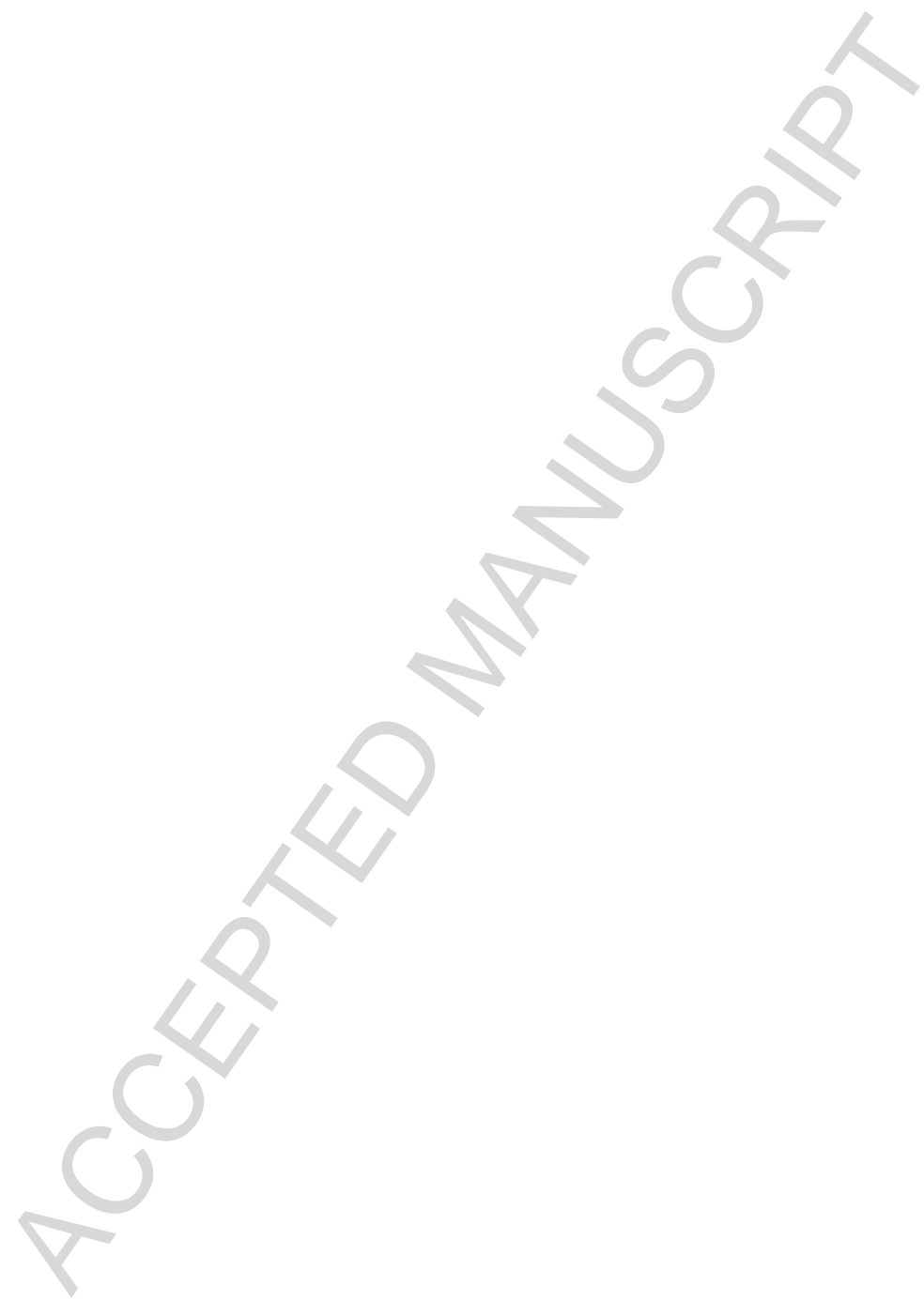




\section{References}

[1] A. Kontush. HDL particle number and size as predictors of cardiovascular disease. Frontiers in Pharmacolog. 6 (2015) 218.

[2] A. Von Eckardstein, G. Assmann. Prevention of coronary heart disease by raising highdensity lipoprotein cholesterol? Curr Opin Lipido. 11 (2000) 627-637.

[3] B.A. Kingwell, M.J. Chapman, A. Kontush, N.E. Miller. HDL-targeted therapies: progress, failures and future. Nature Reviews Drug Discovery. 13 (2014) 445-464.

[4] B.G. Nordestgaard, A. Varbo. Triglycerides and cardiovascular disease. Lancet. 384 (2014) 626-635.

[5] H.K. Siddiqi, D. Kiss, D. Rader. HDL-cholesterol and cardiovascular disease: rethinking our approach. Curr Opin Cardiol. 30 (2015) 536-542.

[6] A.V. Khera, M. Cuchel, M. de la Llera-Moya, A. Rodrigues, M.F. Burke, K. Jafri, B.C. French, J.A. Phillips, M.L. Mucksavage, R.L. Wilensky, E.R. Mohler, G.H. Rothblat, D.J. Rader. Cholesterol efflux capacity, high-density lipoprotein function, and atherosclerosis. $\mathrm{N}$ Engl J Med. 364 (2011) 127-135.

[7] A. Rohatgi, A. Khera, J.D. Berry, E.G. Givens, C.R. Ayers, K.E. Wedin, I.J. Neeland, I.S. Yuhanna, D.R. Rader, J.A. de Lemos, P.W. Shaul. HDL cholesterol efflux capacity and incident cardiovascular events. N Engl J Med. 371 (2014) 2383-2393.

[8] D. Saleheen, R. Scott, S. Javad, W. Zhao, A. Rodrigues, A. Picataggi, D. Lukmanova, M.L. Mucksavage, R. Luben, J. Billheimer, J.J. Kastelein, S.M. Boekholdt, K.T. Khaw, N. Wareham, D.J. Rader. Association of HDL cholesterol efflux capacity with incident coronary heart disease events: a prospective case-control study. Lancet Diabetes \& Endocrinolog. 3 (2015) 507-513.

[9] A. Kontush. HDL-mediated mechanisms of protection in cardiovascular disease. Cardiovasc Res. 103 (2014) 341-349.

[10] J.W. Heinecke. The protein cargo of HDL: implications for vascular wall biology and therapeutics. J Clin Lipidol. 4 (2010) 371-375.

[11] G.H. Rothblat, M.C. Phillips. High-density lipoprotein heterogeneity and function in reverse cholesterol transport. Curr Opin Lipidol. 21 (2010) 229-238.

[12] R.S. Rosenson, H.B. Brewer Jr., W.S. Davidson, Z.A. Fayad, V. Fuster, J. Goldstein, M. Hellerstein, X.C. Jiang, M.C. Phillips, D.J. Rader, A.T. Remaley, G.H. Rothblat, A.R. Tall, L. Yvan-Charvet. Cholesterol efflux and atheroprotection: advancing the concept of reverse cholesterol transport. Circulation. 125 (2012) 1905-1919.

[13] A. Kontush, M.J. Chapman. Antiatherogenic function of HDL particle subpopulations: focus on antioxidative activities. Curr Opin Lipidol. 21 (2010) 312-318.

[14] R. Stocker, J.F. Keaney Jr. Role of Oxidative Modifications in Atherosclerosis. Physiol Rev. 84 (2004) 1381-1478.

[15] X.M. Du, M.J. Kim, L. Hou, W. Le Goff, M.J. Chapman, M. Van Eck, L.K. Curtiss, J.R. Burnett, S.P. Cartland, C.M. Quinn, M. Kockx, A. Kontush, K.A. Rye, L. Kritharides, W. Jessup. HDL particle size is a critical determinant of ABCA1-mediated macrophage cellular cholesterol export. Circ Res. 116 (2015) 1133-1142.

[16] G. Pankhurst, X.L. Wang, D.E. Wilcken, G. Baernthaler, U. Panzenbock, M. Raftery, R. Stocker. Characterization of specifically oxidized apolipoproteins in mildly oxidized high density lipoprotein. J Lipid Res. 44 (2003) 349-355.

[17] B. Garner, P.K. Witting, A.R. Waldeck, J.K. Christison, M. Raftery, R. Stocker. Oxidation of high density lipoproteins. I. Formation of methionine sulfoxide in apolipoproteins AI and All is an early event that accompanies lipid peroxidation and can be enhanced by alphatocopherol. J Biol Chem. 273 (1998) 6080-6087.

[18] A. Zerrad-Saadi, P. Therond, S. Chantepie, M. Couturier, K.A. Rye, M.J. Chapman, A. Kontush. HDL3-Mediated Inactivation of LDL-Associated Phospholipid Hydroperoxides Is 
Determined by the Redox Status of Apolipoprotein A-I and HDL Particle Surface Lipid Rigidity: Relevance to Inflammation and Atherogenesis. Arterioscler Thromb Vasc Biol. 29 (2009) 2169-2175.

[19] A. Kontush, M. Lindahl, M. Lhomme, L. Calabresi, M.J. Chapman, W.S. Davidson. Structure of HDL: particle subclasses and molecular components. Handbook Exp Pharmacol. 224 (2015) 3-51.

[20] L. Camont, M. Lhomme, F. Rached, W. Le Goff, A. Negre-Salvayre, R. Salvayre, C. Calzada, M. Lagarde, M.J. Chapman, A. Kontush. Small, dense high-density lipoprotein-3 particles are enriched in negatively charged phospholipids: relevance to cellular cholesterol efflux, antioxidative, antithrombotic, anti-inflammatory, and antiapoptotic functionalities. Arterioscler Thromb Vasc Biol. 33 (2013) 2715-2723.

[21] E. Nobecourt, S. Jacqueminet, B. Hansel, S. Chantepie, A. Grimaldi, M.J. Chapman, A. Kontush. Defective antioxidative activity of small, dense HDL particles in type 2 diabetes: Relationship to elevated oxidative stress and hyperglycemia. Diabetologia. 48 (2005) 529538.

[22] B. Hansel, A. Kontush, M.T. Twickler. High-density lipoprotein as a key component in the prevention of premature atherosclerotic disease in the insulin resistance syndrome. Seminars in Vascular Medicine. 4 (2004) 215-223.

[23] A. Kontush, M.J. Chapman. Why is HDL functionally deficient in type 2 diabetes? Current Diabetes Reports. 8 (2008) 51-59.

[24] S.J. Nicholls, L. Zheng, S.L. Hazen. Formation of dysfunctional high-density lipoprotein by myeloperoxidase. Trends in Cardiovascular Medicine. 15 (2005) 212-219.

[25] J.W. Heinecke. The role of myeloperoxidase in HDL oxidation and atherogenesis. Current Atherosclerosis Reports. 9 (2007) 249-251.

[26] P.G. Lerch, V. Fortsch, G. Hodler, R. Bolli. Production and characterization of a reconstituted high density lipoprotein for therapeutic applications. Vox Sang. 71 (1996) 155-164.

[27] P.G. Lerch, J.J. Morgenthaler, M. Peitsch, H. Freidli, G. Hodler, J. Doran, H. Isliker, H.J. Heiniger, Isolation and properties of apolipoprotein A for therapeutic use, in: M. Poulik, J.R. Hobbs, J. Mestecky, N. Miller (Eds.), Protides of the Biological Fluids, England: Pergamon Press, Oxford, 1989, pp. 409-416.

[28] C.E. Matz, A. Jonas. Micellar complexes of human apolipoprotein A-I with phosphatidylcholines and cholesterol prepared from cholate-lipid dispersions. J Biol Chem. 257 (1982) 4535-4540.

[29] P. Kistler, H. Nitschmann. Large scale production of human plasma fractions. Vox Sang. 7 (1962) 414-424.

[30] J.W. Brock, A.J. Jenkins, T.J. Lyons, R.L. Klein, E. Yim, M. Lopes-Virella, R.E. Carter, S.R. Thorpe, J.W. Baynes. Increased methionine sulfoxide content of apoA-I in type 1 diabetes. J Lipid Res. 49 (2008) 847-855.

[31] S.C. Rumsey, N.F. Galeano, Y. Arad, R.J. Deckelbaum. Cryopreservation with sucrose maintains normal physical and biological properties of human plasma low density lipoproteins. J Lipid Res. 33 (1992) 1551-1561.

[32] M.J. Chapman, S. Goldstein, D. Lagrange, P.M. Laplaud. A density gradient ultracentrifugal procedure for the isolation of the major lipoprotein classes from human serum. J Lipid Res. 22 (1981) 339-358.

[33] M. Guerin, E. Bruckert, P.J. Dolphin, G. Turpin, M.J. Chapman. Fenofibrate reduces plasma cholesteryl ester transfer from HDL to VLDL and normalizes the atherogenic, dense LDL profile in Combined Hyperlipidemia. Arterioscler Thromb Vasc Biol. 16 (1996) 763-772.

[34] M. Guerin, P. Egger, C. Soudant, W. Le Goff, A. Van Tol, R. Dupuis, M.J. Chapman. Dose-dependent action of atorvastatin in type IIB hyperlipidemia: preferential and 
progressive reduction of atherogenic apoB-containing lipoprotein subclasses (VLDL-2, IDL, small dense LDL) and stimulation of cellular cholesterol efflux. Atherosclerosis.163 (2002) 287-296.

[35] S. Diditchenko, A. Gille, I. Pragst, D. Stadler, M. Waelchli, R. Hamilton, A. Leis, S.D. Wright. Novel formulation of a reconstituted high-density lipoprotein (CSL112) dramatically enhances ABCA1-dependent cholesterol efflux. Arterioscler Thromb Vasc Biol. 33 (2013) 2202-2211.

[36] A. Kontush, S. Chantepie, M.J. Chapman. Small, dense HDL particles exert potent protection of atherogenic LDL against oxidative stress. Arterioscler Thromb Vasc Biol. 23 (2003) 1881-1888.

[37] H. Esterbauer, J. Gebicki, H. Puhl, G. Jurgens. The role of lipid peroxidation and antioxidants in oxidative modification of LDL. Free Radic Biol Med. 13 (1992) 341-390.

[38] H. Zhang, Y. Yang, U.P. Steinbrecher. Structural requirements for the binding of modified proteins to the scavenger receptor of macrophages. J Biol Chem. 268 (1993) 5535-5542.

[39] A. Hafiane, J.K. Bielicki, J.O. Johansson, J. Genest. Novel Apo E-Derived ABCA1 Agonist Peptide (CS-6253) Promotes Reverse Cholesterol Transport and Induces Formation of prebeta-1 HDL In Vitro. PLoS One. 10 (2015) e0131997.

[40] B.J. Van Lenten, M. Navab, D. Shih, A.M. Fogelman, A.J. Lusis. The role of high-density lipoproteins in oxidation and inflammation. Trends in Cardiovascular Medicine. 11 (2001) 155-161.

[41] U. Panzenbock, R. Stocker. Formation of methionine sulfoxide-containing specific forms of oxidized high-density lipoproteins. Biochim Biophys Acta. 1703 (2005) 171-181.

[42] S. Chantepie, E. Malle, W. Sattler, M.J. Chapman, A. Kontush. Distinct HDL subclasses present similar intrinsic susceptibility to oxidation by $\mathrm{HOCl}$. Archives of Biochemistry and Biophysics. 487 (2009) 28-35.

[43] S. Larrede, C.M. Quinn, W. Jessup, E. Frisdal, M. Olivier, V. Hsieh, M.J. Kim, M. Van Eck, P. Couvert, A. Carrie, P. Giral, M.J. Chapman, M. Guerin, W. Le Goff. Stimulation of Cholesterol Efflux by LXR Agonists in Cholesterol-Loaded Human Macrophages Is ABCA1-Dependent but ABCG1-Independent. Arterioscler Thromb Vasc Biol. 29 (2009) 1930-1936.

[44] L. Camont, M.J. Chapman, A. Kontush. Biological activities of HDL subpopulations and their relevance to cardiovascular disease. Trends in Molecular Medicine. 17 (2011) 594603.

[45] K. Curtiss, D.J. Bonnet, K.A. Rye. The conformation of apolipoprotein A-I in high-density lipoproteins is influenced by core lipid composition and particle size: a surface plasmon resonance study. Biochemistry. 39 (2000) 5712-5721.

[46] U. Panzenbock, L. Kritharides, M. Raftery, K.A. Rye, R. Stocker. Oxidation of methionine residues to methionine sulfoxides does not decrease potential antiatherogenic properties of apolipoprotein A-I. J Biol Chem. 275 (2000) 19536-19544.

[47] S. Jayaraman, D.L. Gantz, O. Gursky. Effects of protein oxidation on the structure and stability of model discoidal high-density lipoproteins. Biochemistry. 47 (2008) 3875-3882.

[48] G.M. Anantharamaiah, T.A. Hughes, M. lqbal, A. Gawish, P.J. Neame, M.F. Medley, J.P. Segrest. Effect of oxidation on the properties of apolipoproteins A-I and A-II. J Lipid Res. 29 (1988) 309-318.

[49] S.A. Diditchenko, A. Gille, I. Pragst, D. Stadler, M. Waelchli, R. Hamilton, A. Leis, S.D. Wright. Novel Formulation of a Reconstituted High-Density Lipoprotein (CSL112) Dramatically Enhances ABCA1-Dependent Cholesterol Efflux. Arterioscler Thromb Vasc Biol. 33 (2013) 2202-2211.

[50] A. Gille, R. Easton, D.M. D’Andrea, S.D. Wright, C.L. Shear. CSL112 enhances biomarkers of reverse cholesterol transport after single and multiple infusions in healthy subjects. Arterioscler Thromb Vasc Biol. 34 (2014) 2106-2114. 
[51] P. Tricoci, D.M. D'Andrea, P.A. Gurbel, Zhenling Yao, M. Cuchel, B. Winston, R. Schott, R. Weiss, M.A. Blazing, L. Cannon, A. Bailey, D.J. Angiolillo, A. Gille, C.L. Shear, S.D. Wright, J.H. Alexander. Infusion of Reconstituted High-Density Lipoprotein, CSL112, in Patients With Atherosclerosis: Safety and Pharmacokinetic Results From a Phase 2a Randomized Clinical Trial. J Am Heart Assoc. 4 (2015) e002171.

[52] S.A. Didichenko, A.V. Navdaev, A.M.O. Cukier, A. Gille, P. Schuetz, M.O. Spycher, P. Thérond, M.J. Chapman, A. Kontush, S.D. Wright. Enhanced HDL Functionality in Small HDL Species Produced Upon remodeling of HDL by Reconstituted HDL, CSL112: Effects on Cholesterol Efflux, Anti-Inflammatory and Antioxidative Activity. Circulation Research. 119 (2016) 751-763.

[53] S. Imaizumi, M. Navab, C. Morgantini, C. Charles-Schoeman, F. Su, F. Gao, M. Kwon, E. Ganapathy, D. Meriwether, R. Farias-Eisner, A.M. Fogelman, S.T. Reddy. Dysfunctional high-density lipoprotein and the potential of apolipoprotein A-1 mimetic peptides to normalize the composition and function of lipoproteins. Circulation Journal. 75 (2011) 1533-1538.

[54] S. Tuteja, D.J. Rader. High-density lipoproteins in the prevention of cardiovascular disease: changing the paradigm. Clin Pharmacol Ther 96 (2014) 48-56. 
Highlights

- rHDL inactivates lipid hydroperoxides (LOOH) in a dose- and time-dependent manner

- ApoA-I/lipid ratio is directly related to $\mathrm{LOOH}$-inactivating capacity of $\mathrm{rHDL}$

- ApoA-I/lipid ratio is directly related to $\mathrm{rHDL}$-induced cholesterol efflux via ABCA1

- Oxidation of apoA-I Met112 residue decreases LOOH-inactivating capacity of rHDL

- Oxidation of apoA-I Met112 residue increases cholesterol efflux to rHDL via ABCA1 